\title{
Temperature Dependent IR-Drop Analysis in Graphene Nanoribbon Based Power Interconnect
}

\author{
S. Bhattacharya ${ }^{1, *}$, D. $\operatorname{Das}^{2}$, H. Rahaman ${ }^{1}$ \\ 1 School of VLSI Technology, Indian Institute of Engineering Science and Technology, Shibpur, India \\ ${ }^{2}$ Dept. of Electronics and Communication Engineering, Assam University, Silchar, India
}

(Received 26 December 2015; revised manuscript received 03 March 2016; published online 15 March 2016)

The paper proposes a temperature dependent resistive model of graphene nanoribbon (GNR) based power interconnects. Using the proposed model, IR-drop analysis for $16 \mathrm{~nm}$ technology node latest by ITRS is performed. For a temperature range from $150 \mathrm{~K}$ to $450 \mathrm{~K}$, the variation of resistance of GNR interconnect is $\sim 2-5 \times$ times lesser than that of traditional copper based power interconnects. Our analysis shows that GNR based power interconnects can show $\sim 2-3$ times reduction in Peak IR-drop as compared with copper based interconnects for local, intermediate and global interconnects.

Keywords: Graphene nanoribbon (GNR), Temperature, Peak-IR-Drop, Effective-MFP (mean free path), Interconnect.

PACS numbers: $81.05 . \mathrm{Uw}, \begin{array}{r}63.22 . \mathrm{Np} \\ 61.46 . \mathrm{Np}\end{array}$

\section{INTRODUCTION}

IR-drop has been one of most important challenges of power interconnect in sub-nanometer design [1]. It becomes even more challenging for the high density and high performance designs in which it has adverse effects on timing. The increase in chip operating temperature has two-fold effects on timing. Firstly, it increases the interconnect resistance which in turn increases the interconnect delay. Secondly, due to the increase in resistance there is more IR-drop which also increases the gate delay. Therefore, it is very essential to analyze the effects of temperature on IR-drop in sub nanometer designs, since the resistivity of the traditional copper based interconnects increases significantly in nanometer dimensions [2]. GNR is one of the most promising materials for interconnect modeling for future generation technologies [2,3] due to its excellent properties compared with copper in nanometer dimensions. Recent studies [3-9] on GNR show its superiority over the traditional copper based interconnect. However, to the best of our knowledge no investigation has been performed to analyze the effects of the temperature on IR-drop in GNR interconnect. In this paper, we have proposed a resistive model of graphene nanoribbon (GNR) power interconnect, which is dependent on temperature. Using the proposed model, we have analyzed the IR-drop in GNR based power interconnects. The rest of the paper is organized as follows. Section-II presents the proposed temperature dependent resistive model of GNR interconnect. The results of IR-drop analysis and conclusions are presented in the SectionsIII and IV, respectively.

\section{TEMPERATURE DEPENDENT RESISTANCE MODEL OF GNR INTERCONNECTS}

Due to the presence of large quantum resistance of a monolayer-GNR, a multilayer-GNR structure is proposed for modeling nanointerconnect to utilize the long mean free path as depicted in Fig. 1a. Here, width, thickness, height of multilayer-GNR structure are represented by $w, t, h t$. The separation between two multilayer GNR structures is sp. We have considered $w=16 \mathrm{~nm}$ and $t=32 \mathrm{~nm}$ for $16 \mathrm{~nm}$ ITRS technology node [2]. Fig. $1 \mathrm{~b}$ shows the $2 \mathrm{D}$-honeycomb lattice structure of single layer-GNR.
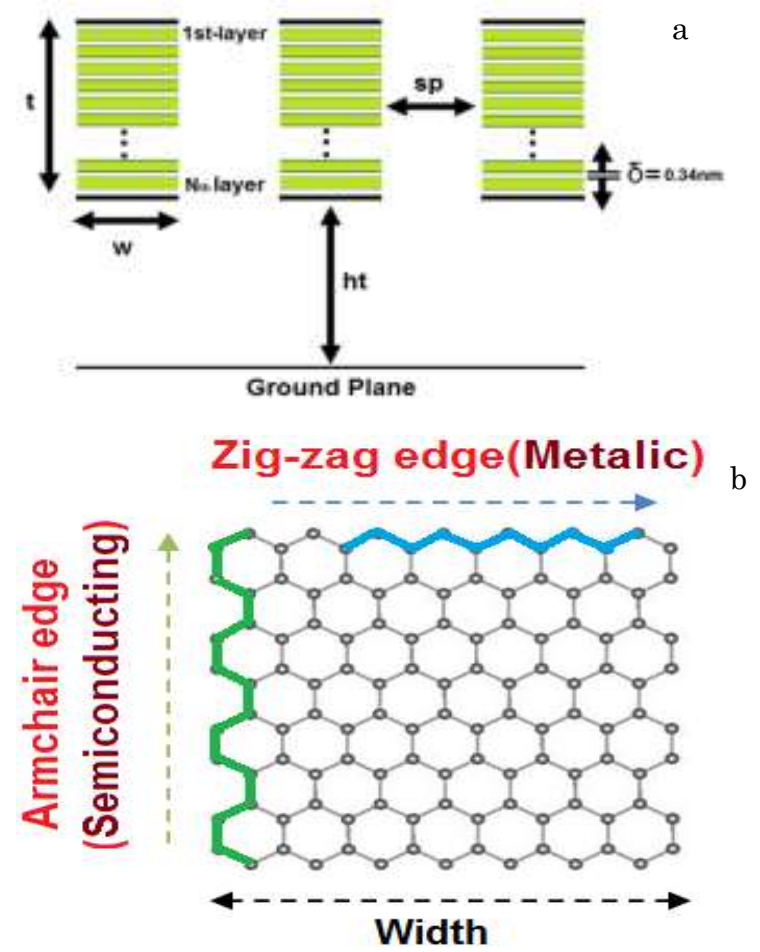

Fig. 1 - Tri-interconnect model of multilayer-GNR structure (a) and 2D honeycomb lattice structure of single layer-GNR (b)

Total number of layers in the multilayer GNR interconnect is reported in as [4]

\footnotetext{
*1983.sandip@gmail.com
} 


$$
N_{\text {layer }}=\left(1+\text { Integer }\left[\frac{t}{\delta}\right]\right)
$$

The spacing ( $\delta$ ) between two graphene layers is $0.34 \mathrm{~nm}$ which is known as van der walls gap [7]. Using (1) we obtain the number of layers as $N_{\text {layer }} \cong 95$ for $16 \mathrm{~nm}$ technology node. The total resistance of MLGNR (multilayer graphene nanoribbon) interconnect is given by [7].

$$
R_{\text {Total-MLGNR }}=R_{Q}\left(1+\frac{l_{M L G N R}}{\lambda_{\text {effective }}}\right)
$$

where $l_{M L G N R}$ is the length of MLGNR based intercon-

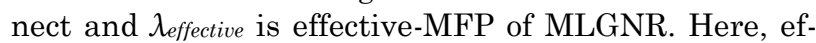
fective-MFP is a function of temperature which is modeled in this section. The quantum resistance $\left(R_{Q}\right)$ is expressed as [4]

$$
R_{Q}=\left[\frac{\left(\frac{h}{2 \cdot e^{2}}\right)}{N_{c h} \cdot N_{\text {layer }}}\right] \Rightarrow\left[\frac{12.94 k \Omega}{N_{c h} \cdot N_{\text {layer }}}\right]
$$

In (3) $N_{c h}$ is the total numbers of conducting channels in monolayer-GNR, $N_{\text {layer }}$ is the number of layers present in multilayer GNR, $h$ is Planck's constant, and $e$ is charge of an electron. The total conducting channels present in monolayer-GNR is expressed in [7].

$$
N_{c h}=\sum_{i=1}^{n_{C}}\left[1+e^{\left(\frac{\left(E_{i, n}-E_{F e}\right)}{k_{B} T}\right)}\right]^{-1}+\sum_{i=1}^{n_{V}}\left[1+e^{\left(\frac{\left(E_{F e}+E_{i, h}\right)}{k_{B} T}\right)}\right]^{-1}
$$

where ' $i$ ' is a positive integer variable, $E_{F e}$ is Fermienergy, $T$ is room temperature, $k_{B}$ is Boltzmann's constant, $n_{c}$ and $n_{v}$ are known as total number of conduction and valance sub-bands. Here, $E_{i, n}$ and $E_{i, h}$ are electron and hole energy for $i^{\text {th }}$ sub-band as expressed as [7]

$$
E_{i}=\frac{i h v_{F}}{2 w}
$$

The total number of channels $\left(N_{c h}\right)$ in metallic GNR is equal to 6 [4-5, 7]. The effective MFP of each layer of GNR depends on three important parameters: electron scattering $\left(\lambda_{e}\right)$, acoustic phonon scattering $\left(\lambda_{a p}\right)$ and remote interfacial phonon scattering $\left(\lambda_{\text {rip }}\right)$. Electron scattering does not depend on the temperature, but the other two parameters vary with temperature. The electron scattering $\lambda_{e}$ can be expressed as [8]

$$
\lambda_{e}=\lambda_{\text {defect }}+w \sum_{a=1}^{N_{c h}} \sqrt{\left(\frac{N_{c h}}{a}\right)-1}
$$

Here, $\lambda_{\text {defect }}$ is a special kind of MFP of graphene. This MFP is due to the defects present in graphene. The value of $\lambda_{\text {defect }}$ is assumed to be $1 \mu \mathrm{m}$ [8]. The acoustic phonon scattering $\left(\lambda_{a p}\right)$ is expressed as [8]

$$
\lambda_{a p}=\frac{h^{2} \rho_{s} v_{s}^{2} v_{f}^{2} w}{\pi^{2} D_{A}^{2} k_{B} T}
$$

In (7), $v_{f}$ is the Fermi velocity of GNR $\left(=8 \times 10^{5} \mathrm{~m} / \mathrm{s}\right), \quad v_{s}$ is the sound velocity of GNR $\left(=2.1 \times 10^{4} \mathrm{~m} / \mathrm{s}\right), \quad D_{A}$ is deformation potential due to acoustic phonon, $k_{B}$ is Boltzmann constants, $\rho_{s}$ is $2 \mathrm{D}$ mass density in graphene, and $T$ is known as room temperature. The remote interfacial phonon scattering can be expressed as [8]

$$
\lambda_{\text {rip }}=\alpha E_{F}^{1.02} W\left(e^{\frac{E_{0}}{k T}}-1\right)
$$

Here, $\alpha$ is the fitting parameter, $E_{F}$ is the Fermi potential $(=0.2 \mathrm{eV})$, and $E_{0}=104 \mathrm{mV}$. The temperature dependent effective MFP of GNR is given by applying Matthiessen's rule [4]

$$
\lambda_{\text {effective }}^{-1}=\lambda_{e}^{-1}+\lambda_{a p}^{-1}+\lambda_{\text {rip }}^{-1}
$$

Substituting the effective MFP of MLGNR in (2) we obtain the temperature dependent resistance of MLGNR.

\section{RESULTS}

Using the temperature dependent resistance model as discussed in previous section, we have calculated the resistance for different interconnect length and different temperature. In Fig. 2 we have shown the temperature dependent resistance of GNR and $\mathrm{Cu}$ interconnect for different interconnect length $(5 \mu \mathrm{m}$ to $50 \mu \mathrm{m})$ for $16 \mathrm{~nm}$ technology node. GNR shows $\sim 2-5 \times$ less resistance than that of $\mathrm{Cu}$ as shown in Fig. 2. With the increase in temperature, the effective mean free path reduces, and hence the scattering induced ohomic part of the total resistance of GNR increases. The IR-drop analysis is performed in GNR and $\mathrm{Cu}$ interconnects for $5 \mu \mathrm{m}$ (local), $20 \mu \mathrm{m}$ (intermediate) and $50 \mu \mathrm{m}$ (global) interconnect lengths. The analysis is per-formed using equivalent circuit model shown in Fig. 3 [9]. In Fig. 3, ten identical CMOS inverters are connected in series with temperature dependent resistance for both $\mathrm{GNR}$ and $\mathrm{Cu}$. In our analysis, we have assumed the supply voltage as $0.7 \mathrm{~V}$, the input-pulse switches from 0 to $0.7 \mathrm{~V}$ for all stages and input-pulse rise and fall time is considered as $100 \mathrm{ps}$.

The CMOS inverters are designed for $16 \mathrm{~nm}$ ITRS technology node using the SPICE models from predictive technology model [10]. The CMOS model parameter for $16 \mathrm{~nm}$ technology as shown in Table 1 . The circuit simulations for CMOS inverter circuit are performed using the Cadence spectre simulator. All the inverters are switched simultaneously so that they draw current from the power supply. As a result the power supply voltage decreases progressively away from the power pad. The decrease in power supply causes increase in propagation delay through the gate. As the temperature increases, the resistance of the power interconnects increases which causes more interconnect delay. With temperature as the IRdrop increases, the gates suffer more delay problem. Therefore, increase in temperature has twofold increase in delay: one due to increase in interconnect (RC) delay and the other due to increase in IR-drop. Figs. 4 through 6 illustrates the IR-drop in GNR and $\mathrm{Cu}$ interconnects for local, intermediate, and global lengths. It is observed that the Peak IR-drop increases with the increase in temperature both for GNR and $\mathrm{Cu}$ interconnects but GNR shows 
$\sim 2$-3× less Peak IR-drop than $\mathrm{Cu}$ at local, intermediate and global lengths.
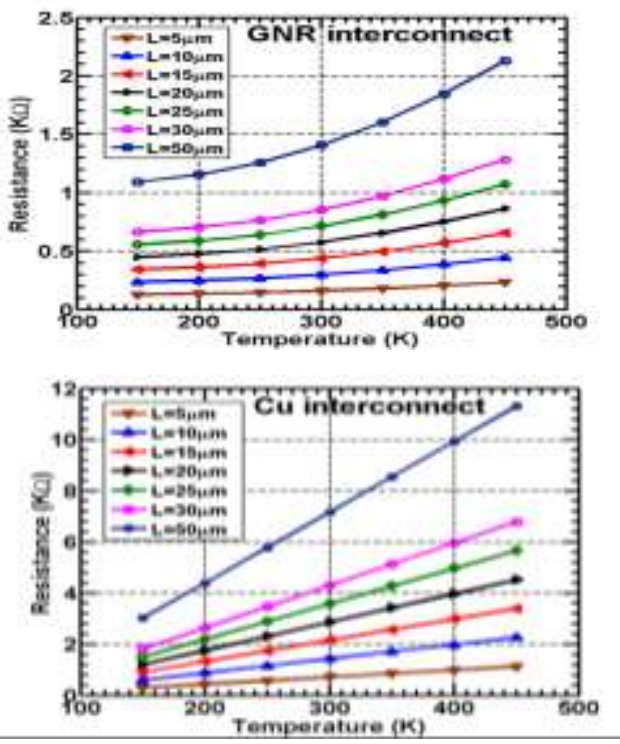

Fig. 2 - Resistance vs. temperature plot for GNR and $\mathrm{Cu}$ interconnect at $16 \mathrm{~nm}$ technology

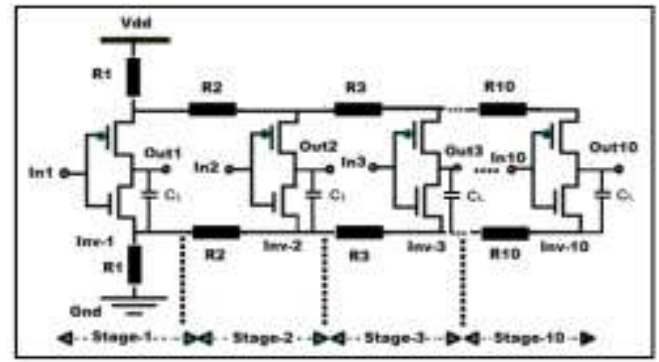

Fig. 3 - Schematic circuit used for power supply voltage drop analysis
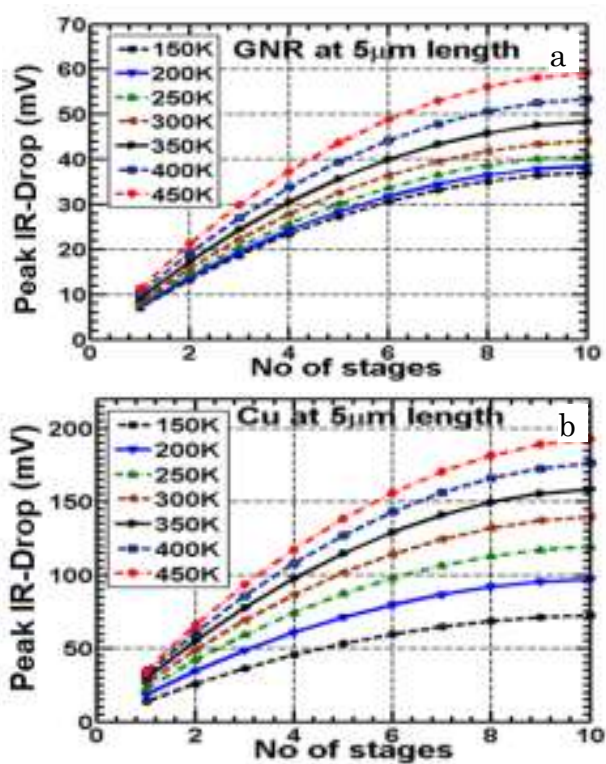

Fig. 4 - Peak IR-drop vs. No of Stages at different temperature for $5 \mu \mathrm{m}$ length for GNR interconnect (local level interconnect) (a) and peak IR-drop vs. No of Stages at different temperature for $5 \mu \mathrm{m}$ length for $\mathrm{Cu}$ interconnect (local level interconnect) (b)
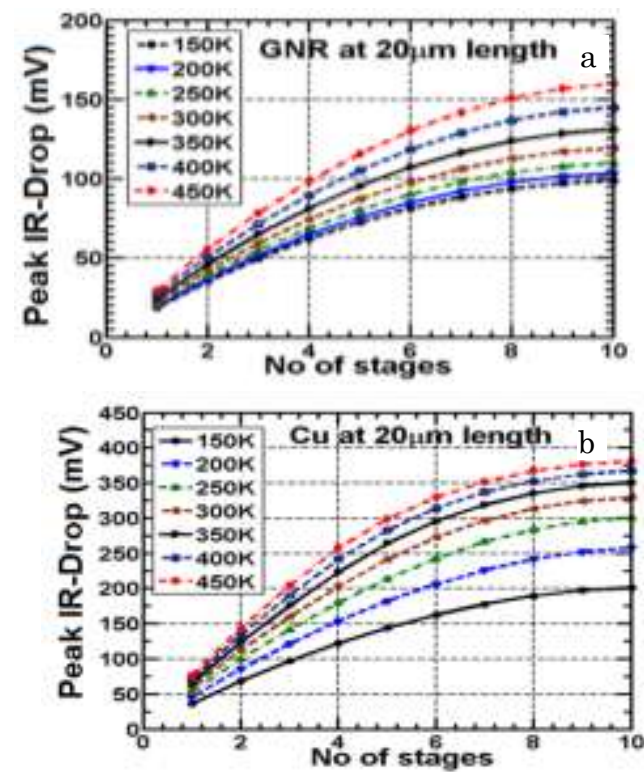

Fig. 5 - Peak IR-drop vs. No of Stages at different temperature for $20 \mu \mathrm{m}$ length for GNR interconnect (intermediate level interconnect) and peak IR-drop vs. No of Stages at different temperature for $20 \mu \mathrm{m}$ length for $\mathrm{Cu}$ interconnect (intermediate level interconnect) (b)
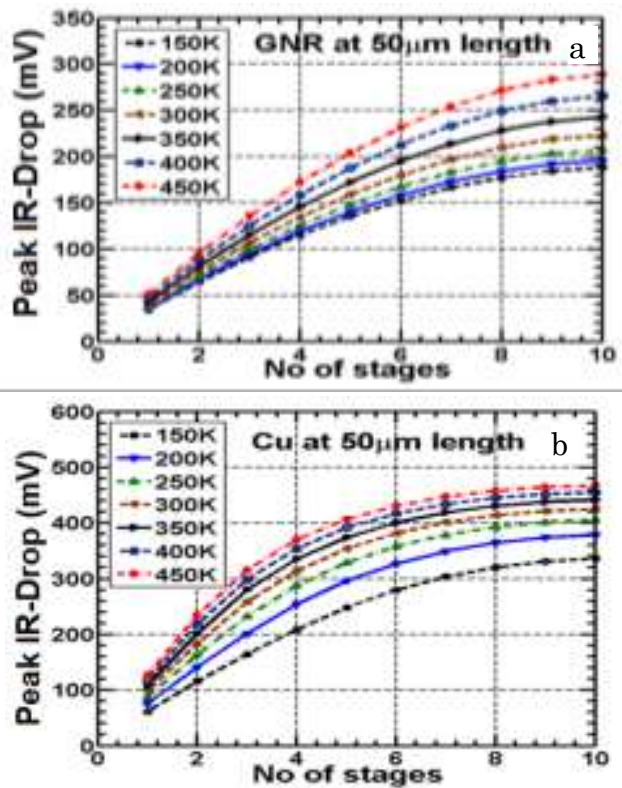

Fig. 6 - Peak IR-drop vs. No of Stages at different temperature for $50 \mu \mathrm{m}$ length for GNR interconnect (global level interconnect) (a) and peak IR-drop vs. No of Stages at different temperature for $50 \mu \mathrm{m}$ length for $\mathrm{Cu}$ interconnect (global level interconnect) (b)

Table 1 - 16 nm PTM CMOS Model Parameters

\begin{tabular}{|c|c|c|}
\hline Model Parameters [10] & $n$-MOS (Si) & $p$-MOS (Si) \\
\hline Channel Length $(L)$ & \multicolumn{2}{|l|}{$16 \mathrm{~nm}$} \\
\hline Channel Width $(W)$ & $64 \mathrm{~nm}$ & $128 \mathrm{~nm}$ \\
\hline Threshold Voltage $\left(V_{\mathrm{TH}}\right)$ & 0.47 volt & -0.43 volt \\
\hline $\begin{array}{l}\text { Dielectric Constant ( } \varepsilon_{0 \times} \text { for } \\
\left.\mathrm{SiO}_{2}\right)\end{array}$ & \multicolumn{2}{|c|}{$\begin{array}{l}\varepsilon_{\text {ox }}=3.9 \times \varepsilon_{0}, \\
\text { Where } \varepsilon_{0}=8.85 \times 10^{-12} \mathrm{~F} / \mathrm{m}\end{array}$} \\
\hline Oxide Thickness $\left(t_{\mathrm{ox}}\right)$ & $0.95 \mathrm{~nm}$ & $1 \mathrm{~nm}$ \\
\hline Gate Oxide Capacitance $\left(C_{\text {ox }}\right)$ & $0.29 \mathrm{fF}$ & $0.28 \mathrm{fF}$ \\
\hline Junction Depth $\left(X_{\mathrm{j}}\right)$ & \multicolumn{2}{|l|}{$5 \mathrm{~nm}$} \\
\hline
\end{tabular}




\section{CONCLUSIONS}

In this work, we have presented a temperature dependent resistive model of GNR interconnect and analyzed the effect of temperature on power supply voltage drop (IR-drop). It is observed that with the increase in temperature, the resistance is increased for both GNR and $\mathrm{Cu}$, but GNR shows significantly less increase than the $\mathrm{Cu}$ interconnects $(\sim 2-5 \times$ times lesser $)$, which exhibits less power supply voltage variation and hence

\section{REFERENCES}

1. A. Vijayakumar, V.C. Patil, G. Paladugu, S. Kundu, 15th International Symposium on Quality Electronic Design (ISQED), 731 (3-5 March, Santa Clara, CA, 2014).

2. International technology roadmap for semiconductors [ITRS-(2007)].

3. A. Naeemi, J.D. Meindl, IITC-2008, 183 (2008).

4. Azad Naeemi, J.D. Meindl, IEEE T. Electron Dev. 56 No 9, 1822 (2009).

5. A. Naeemi, J.D. Meindl, IEEE Electron Dev. Lett. 28 No 5 , 428 (2007).

6. Chuan Xu, Hong Li, Kaustav Banerjee, IEEE T. Electron less impact on the timing of the circuits. It also reduces the power dissipation of GNR based power interconnects as compared with $\mathrm{Cu}$.

\section{AKNOWLEDGEMENTS}

This work is partially supported by the DIT, Government of West Bengal, India under VLSI Design Project.

Dev. $\mathbf{5 6}$ No 8, 1567 (2009)

7. Saeed Haji Nasiri, Mohammad Kazem Moravvej-Farshi, Rahim Faez, IEEE Electron Dev. Lett. 31 No 12, 1458 (2010).

8. S. Tanachutiwat, Shuhong Liu, R. Geer, Wei Wang, IEEE International Symposium on Circuits and Systems, 589 (2009).

9. D. Das, H. Rahaman, 5th International Conference on Computers and Devices for Communication (CODEC), 1 (2012).

10. Predictive Technology Model (2008). 